УДК 517.9

\title{
О работе семинара «Нелинейная динамика»
}

В 2011-2012 годах в рамках научно-образовательного центра «Нелинейная динамика» продолжил работу научный семинар, посвященный исследованиям поведения и методам анализа динамических систем. За прошедший учебный год на нем было заслушано более тридцати сообщений по тематике исследований научно-образовательного центра. Ниже представлены тезисы наиболее интересных докладов, прозвучавших на семинаре.

Нестеров П.Н. Асимптотическое интегрирование систем функциональнодифференциальных уравнений с колебательно убывающими коэффициентами. В докладе исследуется вопрос о построении асимптотики при $t \rightarrow \infty$ решений следующей системы функционально-дифференциальных уравнений:

$$
\begin{aligned}
& \dot{x}=\sum_{i=1}^{n} v_{i}(t) B_{i}\left(t, x_{t}\right)+\sum_{\begin{array}{l}
1 \leq i_{1} \leq i_{2} \leq n \\
\end{array}} v_{i_{1}}(t) v_{i_{2}}(t) B_{i_{1} i_{2}}\left(t, x_{t}\right)+ \\
&+\cdots+\sum_{1 \leq i_{1} \leq \ldots \leq i_{k} \leq n} v_{i_{1}}(t) \cdots v_{i_{k}}(t) B_{i_{1} \ldots i_{k}}\left(t, x_{t}\right)+R\left(t, x_{t}\right) .
\end{aligned}
$$

Здесь $B_{i_{1} \ldots i_{l}}(t, \cdot)$ - ограниченные линейные операторы, действующие из пространства $C_{h} \equiv C\left([-h, 0], \mathbb{C}^{m}\right)$ непрерывных на $[-h, 0]$ вектор-функций со значениями в $\mathbb{C}^{m}$ в пространство $\mathbb{C}^{m}$. Считаем, что в пространстве $C_{h}$ введена стандартная норма

$$
\|\varphi\|=\sup _{-h \leq \theta \leq 0}|\varphi(\theta)| .
$$

Далее, $x \in \mathbb{C}^{m}, x_{t}(\theta)=x(t+\theta)(-h \leq \theta \leq 0)$ - элемент пространства $C_{h}$. Мы предполагаем, что операторы $B_{i_{1} \ldots i_{l}}(t, \cdot)$ являются или периодическими по переменной $t$ с периодом $\omega>0$, т.е.

$$
B_{i_{1} \ldots i_{l}}(t+\omega, \varphi) \equiv B_{i_{1} \ldots i_{l}}(t, \varphi), \quad \varphi \in C_{h},
$$

или допускают представление в виде

$$
B_{i_{1} \ldots i_{l}}(t, \varphi)=\sum_{j=1}^{N} \Gamma_{j}^{\left(i_{1} \ldots i_{l}\right)}(t) \ell_{j}^{\left(i_{1} \ldots i_{l}\right)}(\varphi), \quad \varphi \in C_{h}
$$

В формуле $(3), \ell_{j}^{\left(i_{1} \ldots i_{l}\right)}(\varphi)$ - ограниченные линейные операторы, действующие из $C_{h}$ в $\mathbb{C}^{m}$ и не зависящие от $t$, и $\Gamma_{j}^{\left(i_{1} \ldots i_{l}\right)}(t)$ - некоторые матрицы, элементами которых являются тригонометрические многочлены, т.е.

$$
\Gamma_{j}^{\left(i_{1} \ldots i_{l}\right)}(t)=\sum_{s=1}^{M} \beta_{s j}^{\left(i_{1} \ldots i_{l}\right)} e^{\mathfrak{i} \lambda_{s} t}
$$


Здесь $\beta_{s j}^{\left(i_{1} \ldots i_{l}\right)}$ - постоянные квадратные матрицы и $\lambda_{s}-$ действительные числа. Если выполнено тождество (2), то мы будем предполагать, что для каждого $\varphi \in C_{h}$ функции $B_{i_{1} \ldots i_{l}}(\cdot, \varphi)$ измеримы по Лебегу и для всех $\varphi \in C_{h}$ и $t \in \mathbb{R}$

$$
\left|B_{i_{1} \ldots i_{l}}(t, \varphi)\right| \leq K\|\varphi\|,
$$

где $K$ - некоторая постоянная. Очевидно, что неравенство (5) оказывается выполненным и в том случае, когда операторы $B_{i_{1} \ldots i_{l}}(t, \varphi)$ имеют вид $(3),(4)$.

Наконец, $R(t, \cdot)\left(t \geq t_{0}\right)$ - линейный ограниченный оператор, действующий из $C_{h}$ в $\mathbb{C}^{m}$, относительно которого предполагается следующее. Для любого $\varphi \in C_{h}$, вектор-функция $R(\cdot, \varphi)$ измерима по Лебегу при $t \geq t_{0}$, и, кроме того, существует скалярная функция $\gamma(t) \in L_{1}\left[t_{0}, \infty\right)$, такая что

$$
|R(t, \varphi)| \leq \gamma(t)\|\varphi\|, \quad \varphi \in C_{h}, \quad t \geq t_{0} .
$$

Осталось заметить, что $v_{1}(t), \ldots, v_{n}(t)$ - абсолютно непрерывные комплекснозначные скалярные функции, заданные на интервале $\left[t_{0}, \infty\right)$, относительно которых предполагается, что

$$
\begin{array}{ll}
1^{0} . & v_{1}(t) \rightarrow 0, v_{2}(t) \rightarrow 0, \ldots, v_{n}(t) \rightarrow 0 \text { при } t \rightarrow \infty ; \\
2^{0} . & \dot{v}_{1}(t), \dot{v}_{2}(t), \ldots, \dot{v}_{n}(t) \in L_{1}\left[t_{0}, \infty\right) ; \\
3^{0} . & v_{i_{1}}(t) v_{i_{2}}(t) \cdots v_{i_{k+1}}(t) \in L_{1}\left[t_{0}, \infty\right) \text { для любых } 1 \leq i_{1} \leq i_{2} \leq \cdots \leq i_{k+1} \leq n .
\end{array}
$$

Известно, что при сформулированных условиях для любой функции $\varphi \in C_{h}$ и любого $T \geq t_{0}$ существует единственная функция $x(t)$, которая удовлетворяет (1) при $t \geq T$ с начальным условием $x_{T}=\varphi$ (см., например, [1]).

Используя вариант метода усреднения, предложенный в работе [2], систему (1) удается привести к некоторому специальному виду. Затем для построения асимптотики решений полученной системы используется аналог асимптотической теоремы Н. Левинсона [3].

В качестве иллюстрации предложенной методики в докладе получены асимптотические формулы для решений дифференциального уравнения с запаздыванием

$$
\ddot{x}+x+\frac{a \sin \lambda t}{t^{\rho}} x(t-h)=0,
$$

где $a, \lambda \in \mathbb{R}, \rho>0$ и $h>0$.

1. Хейл Дж. Теория функционально-дифференциальных уравнений. М.: Мир, 1984.

2. Нестеров П.Н. Метод усреднения в задаче асимптотического интегрирования систем с колебательно убывающими коэффициентами // Дифференциальные уравнения. 2007. T. 43, №6. С. 731-742.

3. Cassel J.S., Hou Z. Asymptotically diagonal linear differential equations with retardation // J. Lond. Math. Soc. 1993. V. 47. P. 473-483.

Малозёмова Д.В. Высокомодовые аттракторы в модификации уравнения Свифта-Хоэнберга. Уравнением Свифта-Хоэнберга принято называть нелинейное параболическое уравнение вида

$$
\partial_{t} w=\varepsilon w-(1+\Delta)^{2} w-w^{3} .
$$


Ограничимся ситуацией, когда $w$ - действительная функция от $(x, t) \in[0, \pi] \times R$, а $\varepsilon-$ малый положительный параметр. Это уравнение впервые было предложено в статье [1] как простейшая феноменологическая модель конвекции Релея-Бенара. В дальнейшем оно широко использовалось в качестве модельного в различных областях естествознания.

Рассмотрим модификацию уравнения (1)

$$
\partial_{t} w=\varepsilon w-\left(1+\nu \partial_{x}^{2}\right)^{2} w-w \partial_{x} w
$$

дополненную краевыми условиями типа Дирихле:

$$
\left.w\right|_{x=0, \pi}=\left.\partial_{x}^{2} w\right|_{x=0, \pi}=0 .
$$

Для задачи (2), (3) можно сформулировать результат о формировании в ней высокомодовых аттракторов.

Линейный анализ системы показывает, что при $\nu \in \bigcup_{n=1}^{+\infty}\left(\frac{\nu_{-}(\varepsilon)}{n^{2}}, \frac{\nu_{+}(\varepsilon)}{n^{2}}\right), \nu_{ \pm}(\varepsilon)=1 \pm$ $\sqrt{\varepsilon}$ нулевое решение становится неустойчивым. В связи с этим нас будут интересовать возникающие в системах неоднородные стационарные решения, рождающиеся из нуля при $\nu=\nu_{-}(\varepsilon) / n^{2}$ и исчезающие в нем при $\nu=\nu_{+}(\varepsilon) / n^{2}$. Рассмотрим случай $n=1$.

Для нахождения существующих состояний равновесия будем считать, что

$$
0<\varepsilon<<1, \quad \nu=1+\delta \sqrt{\varepsilon}, \quad \delta \in(-1,1),
$$

где параметр $\delta$, имеющий порядок единицы, отвечает за изменение $\nu$ на требуемом интервале $\left(\nu_{-}(\varepsilon), \nu_{+}(\varepsilon)\right)$.

Теорема 1. При выполнении условий (4) краевая задача (1),(2) имеет две экспоненицально устойчивые диссипативные структуры:

$$
w_{1}(x, \delta, \varepsilon)=w_{0}(x, \delta, \varepsilon), w_{2}(x, \delta, \varepsilon)=w_{0}(\pi-x, \delta, \varepsilon), w_{0}(x,-1, \varepsilon)=w_{0}(x, 1, \varepsilon) \equiv 0,
$$

где функиия $w_{0}(x, \delta, \varepsilon)$ допускает равномерное по $\delta \in\left[\delta_{1}, \delta_{2}\right] \subset(-1,1)$ асимптотическое представление

$$
w=\sqrt{\varepsilon} w_{1}(x)+\varepsilon w_{2}(x)+\varepsilon^{3 / 2} w_{3}(x)+\ldots, \quad w_{1}(x)=\xi_{0} \sin x,
$$

а $\xi_{0}>0$ однозначно определяется в ходе вычислений.

На основании описанного в [2] принципа подобия, из найденного решения можно сконструировать и все остальные рождающиеся из нуля неоднородные стационарные решения, возникающие при $\nu \in\left(\nu_{n}^{-}(\varepsilon), \nu_{n}^{+}(\varepsilon)\right)$.

Теорема 2. Найдется такое достаточно малое $\varepsilon_{0} \in(0,1)$, что при любом фиксированном $\varepsilon \in\left(0, \varepsilon_{0}\right]$ и при каждом натуральном $n$ на соответствующем интервале $\left(\nu_{n}^{-}(\varepsilon), \nu_{n}^{+}(\varepsilon)\right)$ изменения параметра $\nu$ каждая из краевых задач (1), (2) имеет пару диссипативных структур

$$
\begin{aligned}
& w_{n}^{1}(x, \nu, \varepsilon)=\frac{1}{n} w_{0}\left(n x, \delta_{n}(\nu, \varepsilon), \varepsilon\right), w_{n}^{2}(x, \nu, \varepsilon)=\frac{1}{n} w_{0}\left(\pi-n x, \delta_{n}(\nu, \varepsilon), \varepsilon\right), \\
& \text { где } \delta_{n}(\nu, \varepsilon)=\left(n^{2} \nu-1\right) / \sqrt{\varepsilon} .
\end{aligned}
$$

Фиксируем произвольно два числа $\delta_{1}$ и $\delta_{2}$ из интервала $(-1 / \sqrt{3}, 1 / \sqrt{3})$. На вопрос об устойчивости найденных в теоремах 1 и 2 решений отвечает следующая теорема.

Теорема 3. Найдется такое достаточно малое $\varepsilon_{0}=\varepsilon_{0}\left(\delta_{1}, \delta_{2}\right) \in(0,1)$, что при всех $0<\varepsilon \leq \varepsilon_{0}$ и при каждом $n \geq 1$ пара диссипативных структур (7), (8) краевых задач 
(1), (2) экспоненциально устойчива на отрезке $\left[\left(1+\delta_{1} \sqrt{\varepsilon}\right) / n^{2},\left(1+\delta_{2} \sqrt{\varepsilon}\right) / n^{2}\right]$ изменения параметра $\nu$.

Поскольку множество $\bigcup_{n=1}^{\infty}\left(\nu_{n}^{-}(\varepsilon), \nu_{n}^{+}(\varepsilon)\right)$ устроено так, что с уменьшением $\nu$ интервалы пересекаются в неограниченно возрастающем количестве, то в системах (1), (2) происходит неограниченное накопление устойчивых неоднородных стационарных решений, то есть реализуется феномен буферности (см. [2]).

В ходе численного моделирования было установлено, что указанные в теоремах 1 и 2 стационарные решения являются не единственными аттракторами. Благодаря квадратичному типу нелинейности, система обладает достаточно богатой глобальной динамикой. Она демонстрирует другую серию устойчивых стационаров, а также устойчивые периодические решения, которые при изменении параметров демонстрируют бифуркации удвоения периода. Особенности глобальной динамики представляется возможным осветить с помощью более подробного численного исследования.

Работа выполнена при финансовой поддержке гранта Правительства РФ по постановлению № 220, договор № 11.G34.31.0053.

1. Swift J., Hohenberg P.S. Hydrodynamic fluctuations at the convective instability // Phys. Rev. 1977. V. A15, №1. P. 319-328.

2. Мищенко Е.Ф., Садовничий В.А., Колесов А.Ю., Розов Н.Х. Автоволновые процессы в нелинейных средах с диффузией. М.: ФИЗМАТЛИТ, 2005. 432 с.

Бобок А.С. Локальный анализ цепочки автогенераторов в опыте Скотта. Рассматривается дискретная математическая модель цепочки $N$ связанных нелинейных автогенераторов, взятая из статьи [1]

$$
\frac{\partial^{2} u_{k}}{\partial t^{2}}-\varepsilon \frac{\partial u_{k}}{\partial t}-\varepsilon \nu \frac{\partial}{\partial t} L u_{k}+u_{k}=L u_{k}-\varepsilon u_{k}^{2} \frac{\partial u_{k}}{\partial t}, \quad k=1, \ldots, N .
$$

Здесь $L u_{k}$ - разностный оператор вида $L u_{k}=\delta^{2}\left(u_{k+1}-2 u_{k}+u_{k-1}\right), \varepsilon-$ малый параметр. Условия на границе взяты имеющими вид

$$
u_{0}=0, \quad u_{N+1}=0 .
$$

Исследованы варианты поведения системы, как в безрезонансном случае, так и в случае резонансов специального вида, были получены соответствующие нормальные формы и проведен локальный анализ устойчивости состояний равновесия системы. Ниже приведена нормальная форма, построенная в предположении отсутствия резонансов

$$
2 \frac{d z_{n}}{d \tau}=\left(1-\nu\left(\omega_{n}^{2}-1\right)\right) z_{n}-\frac{3}{4} z_{n}\left|z_{n}\right|^{2}-\sum_{k=1, k \neq n}^{N} z_{n}\left|z_{k}\right|^{2}, \quad n=1, \ldots, N,
$$

где $\omega_{n}=\sqrt{1+2 \delta^{2}\left(1-\cos \frac{\pi n}{N+1}\right)}, \quad n=1, \ldots, N$.

Сформулируем теорему о сосуществовании в исходной системе максимального числа устойчивых однокомпонентных режимов (см. аналогичные утверждения в $[2,3]$ ).

Теорема. Пусть для краевой задачи (1) - (2) выполнено условие

$$
\begin{aligned}
& \omega_{N}^{2}<\frac{1+\nu}{\nu}, \\
& 1-\nu\left(\omega_{1}^{2}-1\right)<\frac{4}{3}\left(1-\nu\left(\omega_{N}^{2}-1\right)\right),
\end{aligned}
$$


где $\omega_{1}, \omega_{N}$ заданы выше, тогда существует такое $\varepsilon_{0}>0$, что для всех $0<\varepsilon<\varepsilon_{0}$ задача (1) - (2) имеет максимальное количество (ровно N) сосуществующих орбитально асимптотически устойчивых чиклов, асимптотика которых задается следующей формулой:

$$
u_{i}(t, j)=\frac{4 \sqrt{3}}{3} \sqrt{1-\nu\left(\omega_{i}^{2}-1\right)} \cos \left(\omega_{i} t+\varphi_{i}\right) \sin \frac{\pi i j}{N+1}+O(\varepsilon), \quad i=1, \ldots, N .
$$

Заметим при этом, что все остальные режимы исходной системы с числом ненулевых компонент, большим единицы, неустойчивы.

1. Колесов А.Ю., Розов Н.Х., Садовничий В. А. Математические аспекты теории развития турбулентности по Ландау // УМН. 2008. 63:2(380). С. 21--84.

2. Глызин С. Д., Колесов А.Ю., Розов Н.Х. К вопросу о реализуемости сценария развития турбулентности по Ландау // ТМФ. 2009. 158:2. С. 292--311.

3. Глызин С.Д., Колесов А. Ю., Розов Н.Х. Хаотическая буферность в цепочках связанных осцилляторов // Дифференциальные уравнения. 2005. Т. 41, №1. С. 41-49.

Кубышкин Е.П. Метод равномерной нормализации в исследовании периодических решений дифференциально-разностных уравнений с малым параметром при производной.

Рассматривается дифференциально-разностное уравнение вида

$$
\varepsilon_{1} \dot{x}(t)+x(t)+f(x(t-1))=0,
$$

где $0<\varepsilon_{1}<<1, f(x) \equiv-f(-x)$ - нелинейная функция класса $C^{\infty}, f(x)=f_{1} x+f_{3} x^{3}+$ $o\left(|x|^{3}\right)\left(|x|<x_{0}, f_{1}>0, f_{3}<0\right)$. Уравнения вида (1) возникают при изучении многих прикладных задач. В предположении $f_{1}=1+\varepsilon_{2},\left|\varepsilon_{2}\right|<<1$ изложен метод равномерной по $\varepsilon\left(\varepsilon=\left(\varepsilon_{1}, \varepsilon_{2}\right),|\varepsilon|<\varepsilon_{0},|\varepsilon|=\left(\varepsilon_{1}^{2}+\varepsilon_{2}^{2}\right)^{1 / 2}\right)$ нормализации уравнения $(1)$, с помощью которого изучаются его периодические решения, возникающие из нулевого состояния при изменении параметров и принадлежащие некоторой фиксированной окрестности нулевого решения.

Введем в рассмотрение характеристическое уравнение

$$
P\left(\lambda ; \varepsilon_{1}\right) \equiv \varepsilon_{1} \lambda+1+\left(1+\varepsilon_{2}\right) \exp (-\lambda)=0
$$

линейной части уравнения (1).

Утверждение 1. Множество $\left\{\lambda_{n}(\varepsilon), \lambda_{-n}(\varepsilon)=\bar{\lambda}_{n}(\varepsilon), n=1,3,5, \ldots\right\}$, где $\lambda_{n}(\varepsilon)=i \pi n+$ $\ln \left(1+\varepsilon_{2}\right)+\lambda^{1}\left(i \pi n+\ln \left(1+\varepsilon_{2}\right) ; \varepsilon_{1}\right)=i \pi n+\ln \left(1+\varepsilon_{2}\right)-\ln \left(1+\varepsilon_{1}\left(i \pi n+\ln \left(1+\varepsilon_{2}\right)\right)\right)+$ $o(|\varepsilon|)$, определяет совокупность корней характеристического уравнения. Здесь $\lambda^{1}\left(w ; \varepsilon_{1}\right) \equiv$ $-\ln \left(1+\varepsilon_{1}\left(w-\ln \left(1+\varepsilon_{1}\left(w-\ln \left(1+\varepsilon_{1}(w-\ldots)\right)\right)\right)\right)\right)$, второе равенство в определении $\lambda_{n}(\varepsilon)$ выполняется равномерно относительно $n$.

Обозначим через $l_{2}$ и $l_{2}^{1}\left(l_{2}^{1} \subset l_{2}\right)$ пространства последовательностей вида $z=\left(z_{1}, z_{3}, z_{5}, \ldots\right), z_{j} \in C$, удовлетворяющих условиям $\|z\|_{l_{2}}^{2}=\sum_{n=1}^{\infty}\left|z_{n}\right|^{2}<\infty$ и $\|z\|_{l_{2}^{1}}^{2}=$ $\sum_{n=1}^{\infty}\left|z_{n} \lambda_{n}(\varepsilon)\right|^{2}<\infty$ соответственно, $s\left(r_{0}\right)=\left\{z:\|z\|_{l_{2}^{1}}^{2}<r_{0}\right\}$ и введем в рассмотрение систему обыкновенных дифференциальных уравнений в пространстве $l_{2}$ вида:

$$
\dot{z_{n}}=\lambda_{n}(\varepsilon) z_{n}+Z_{n}^{(3)}(z, \bar{z} ; \varepsilon), \quad\left(Z_{n}^{(3)}(z, \bar{z} ; \varepsilon) \equiv \sum_{\left(n_{1} n_{2} n_{3}\right) \in \Omega_{n}^{3}} d_{n_{1} n_{2} n_{3}}(\varepsilon) z_{n_{1}} z_{n_{2}} z_{n_{3}}\right),
$$


в которой $(n= \pm 1, \pm 3, \ldots), \Omega_{n}^{3}=\left\{\left(n_{1}, n_{2}, n_{3}\right): n_{j}= \pm 1, \pm 3 \ldots, n=n_{1}+n_{2}+n_{3}\right\}$, $z_{-n}=\overline{z_{n}}, z \in s\left(r_{0}\right), d_{-n_{1}-n_{2}-n_{3}}=\overline{d_{n_{1} n_{2} n_{3}}}, d_{n_{1} n_{2} n_{3}}(\varepsilon)=\left[\varepsilon_{1}+\left(1+\varepsilon_{2}\right) \exp \left(-\lambda_{n_{1} n_{2} n_{3}}(\varepsilon)\right)(1-\right.$ $\left.\exp \left(\lambda_{n_{1} n_{2} n_{3}}(\varepsilon)-\lambda_{n}(\varepsilon)\right) /\left(\lambda_{n_{1} n_{2} n_{3}}(\varepsilon)-\lambda_{n}(\varepsilon)\right)\right]^{-1} p f_{3} \exp \left(-\lambda_{n_{1} n_{2} n_{3}}(\varepsilon)\right) \cdot\left(1+\varepsilon_{1}+\varepsilon_{1} \lambda_{n}\right)^{1 / 2}(1+$ $\left.\varepsilon_{1}+\varepsilon_{1} \lambda_{n_{1}}\right)^{-1 / 2} \cdot\left(1+\varepsilon_{1}+\varepsilon_{1} \lambda_{n_{2}}\right)^{-1 / 2}\left(1+\varepsilon_{1}+\varepsilon_{1} \lambda_{n_{3}}\right)^{-1 / 2}$. Параметр $p$ принимает значение 1,2 или 3 в зависимости от вида резонанса. Систему уравнений (2) будем называть равномерной нормальной формой уравнения (1). Перейдем в (2) к полярным координатам, положив $z_{n}=\rho_{n} \exp \left(i \tau_{n}\right)\left(\rho_{n} \geq 0\right)$. Структура системы уравнений $(2)$ такова, что позволяет ввести одну „быструю“ переменную $\tau_{1}$, для которой имеем дифференциальное уравнение $\dot{\tau_{1}}=\pi+\sigma_{1}(\varepsilon)+T(\rho, \theta ; \varepsilon)$, и счетное число „медленных“ переменных $\rho=\left(\rho_{1}, \rho_{3}, \rho_{5}, \ldots\right)$, $\theta=\left(\theta_{1}, \theta_{3}, \theta_{5}, \ldots\right)\left(\theta_{j} \in[0,2 \pi)\right)$, для которых имеем следующую систему дифференциальных уравнений:

$$
\dot{\rho_{n}}=\gamma_{n}(\varepsilon) \rho_{n}+R_{n}(\rho, \theta ; \varepsilon), \quad \dot{\theta_{n}}=\delta_{n}(\varepsilon)+\Theta_{n}(\rho, \theta ; \varepsilon),(n=1,3,5, \ldots) .
$$

В (3) функционалы $R_{n}(\rho, \tau ; \varepsilon), \Theta_{n}(\rho, \theta ; \varepsilon)$ и функционал $T(\rho, \tau ; \varepsilon)$ гладко зависят от своих переменных и $2 \pi$ периодические по $\theta_{j}(j=1,3,5, \ldots), \lambda_{n}(\varepsilon)=\gamma_{n}(\varepsilon)+i \sigma_{n}(\varepsilon), \delta_{n}(\varepsilon)(\delta(0)=0)$ является конечной линейной комбинацией из $\sigma_{j}(\varepsilon)$.

Предположим, что в области $|\varepsilon| \leq \varepsilon_{0}$ имеется подобласть $\Omega\left(\varepsilon_{0}\right)$, примыкающая к точке $\varepsilon=0$, при значениях $\varepsilon$ из которой система уравнений (3) имеет экспоненциально устойчивое состояние равновесия $\left(\rho^{*}(\varepsilon), \theta^{*}(\varepsilon)\right),\left(\rho^{*}(\varepsilon) \rightarrow 0, \theta^{*}(\varepsilon) \rightarrow \theta^{*}(0) \in[0 ; 2 \pi)\right.$ при $\left.\varepsilon \rightarrow 0, \rho^{*}(\varepsilon) \in l_{2}^{1}\right)$. Обозначим $\rho=\rho(\varepsilon)=\left\|\rho^{*}(\varepsilon)\right\|_{l^{1}}=\left(\sum_{j=1}^{\infty} \rho_{j}^{* 2}(\varepsilon)\left|\lambda_{j}(\varepsilon)\right|^{2}\right)^{1 / 2}, \xi_{n}^{*}(\varepsilon)=\rho_{n}^{*}(\varepsilon) / \rho$ и введем фазу синхронизации $\alpha_{n}^{*}(\varepsilon)=\tau_{n}-n \tau_{1}$, вычисленную на указанном состоянии равновесия.

Утверждение 2. Пусть при $\varepsilon \in \Omega\left(\varepsilon_{0}\right)$ для некоторого $\varepsilon_{0}$ система уравнений (3) имеет состояние равновесия с перечисленными выше свойствами. Тогда при указанных значениях $\varepsilon$ уравнение (1) имеет асимптотически орбитально устойчивый предельный цикл, период которого $T(\varepsilon) \rightarrow 2$ при $\varepsilon \rightarrow 0$ и имеющий следующую асимптотическую формулу:

$$
\begin{aligned}
& x^{*}(t ; \varepsilon)=\rho x_{1}^{*}\left(\tau_{1} ; \varepsilon\right)+\rho^{3} x_{3}^{*}\left(\tau_{1} ; \rho, \varepsilon\right), \quad x_{1}^{*}\left(\tau_{1} ; \varepsilon\right)=\sum_{-\infty}^{\infty} \xi_{n}^{*}(\varepsilon) \exp \left(i\left(n \tau_{1}+\alpha_{n}^{*}(\varepsilon)\right)\right), \\
& \dot{\tau_{1}}=\pi+\sigma_{1}(\varepsilon)+\rho^{2} \sigma_{2}^{*}(\rho, \varepsilon),
\end{aligned}
$$

где функции $x_{3}^{*}\left(\tau_{1} ; \rho, \varepsilon\right) \equiv x_{3}^{*}\left(\tau_{1}+2 \pi ; \rho, \varepsilon\right)$ и $\sigma_{2}^{*}(\rho, \varepsilon)$ непрерывны по совокупности переменных и бесконечно дифференцируемы по $\tau_{1}, \rho$.

Глызин С. Д., Горчакова Е. В. Слабое взаимодействие двух не идентичных уравнений Хатчинсона. Рассматривается система двух слабосвязанных логистических уравнений с запаздыванием

$$
\begin{aligned}
& \dot{N}_{1}=r_{1}\left[1-N_{1}(t-1)\right] N_{1}+D\left(N_{2}-N_{1}\right), \\
& \dot{N}_{2}=r_{2}\left[1-N_{2}(t-h)\right] N_{2}+D\left(N_{1}-N_{2}\right),
\end{aligned}
$$

находящая приложения в популяционной динамике. Локальный анализ (1) выполняется при условии, что параметры задачи близки к критическому случаю двух пар чисто мнимых собственных значений. Ниже считаем

$$
r_{1}=\frac{\pi}{2}+\varepsilon, r_{2}=\frac{\pi}{2}+\alpha \varepsilon, h=1+\varepsilon h_{0}, D=\varepsilon d,
$$


где $\varepsilon$ - положительный малый параметр, а величины $\alpha, h_{0}, d$ имеют порядок единицы. Отметим, что параметр связи $d>0$, а знак $\alpha$ и $h_{0}$, вообще говоря, произволен.

Для применения метода нормальных форм в окрестности состояния равновесия $N_{1}=$ $N_{2} \equiv 1$ выполним стандартную замену Пуанкаре-Боголюбова-Митропольского

$$
N_{j}(t, s)=1+\sqrt{\varepsilon} u_{0 j}(t, s)+\varepsilon u_{1 j}(t, s)+\varepsilon^{3 / 2} u_{2 j}(t, s)+\ldots \quad j=1,2,
$$

где $s=\varepsilon t-$ медленное время, $u_{0 j}(t, s)=z_{j}(s) \exp (i \pi t / 2)+\bar{z}_{j}(s) \exp (-i \pi t / 2)$, а $u_{1 j}(t, s)$ и $u_{2 j}(t, s)-4$-периодические по $t$ функции. Медленно меняющиеся комплексные амплитуды $z_{1}(s)$ и $z_{2}(s)$ определяются из условий разрешимости задач для $u_{2 j}(t, s)(j=1,2)$ в классе 4 -периодических по $t$ функций. Система

$$
\begin{aligned}
& z_{1}^{\prime} \cdot\left(1+i \frac{\pi}{2}\right)=i z_{1}+\frac{\pi(-1+3 i)}{5}\left|z_{1}\right|^{2} z_{1}+d\left(z_{2}-z_{1}\right) \\
& z_{2}^{\prime} \cdot\left(1+i \frac{\pi}{2}\right)=\left(\frac{\pi^{2}}{4} h_{0}+\alpha i\right) z_{2}+\frac{\pi(-1+3 i)}{5}\left|z_{2}\right|^{2} z_{2}+d\left(z_{1}-z_{2}\right),
\end{aligned}
$$

представляет собой нормальную форму системы (1) при условиях (2) с отброшенными членами более высокого по $\varepsilon$ порядка малости. Штрихом в (4) обозначена производная функций $z_{j}(s)$ по $s$. Для нормальной формы выполнена стандартная теорема о соответствии, состоящая в том, что ее экспоненииально устойчивым или дихотомичным авто-

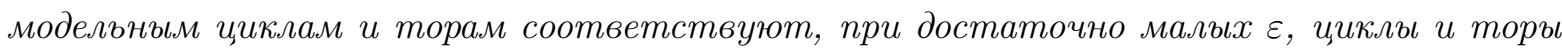
исходной системы (1) той же устойчивости. Представляет интерес подробное исследование фазовых перестроек (4) при изменении параметра связи $d$, а также выявление изменений бифуркационных сценариев при изменении параметров $\alpha$ и $h_{0}$.

Отметим, что система (4) исследовалась в [1] при $\alpha=1$ и $h_{0}=0$, а в статье [2] - в случае связи конкурентного типа, наконец, в статьях [3] и [4] аналогичная задача решалась для пары осцилляторов из нейродинамики.

1. Глызин С. Д. Сценарии фазовых перестроек одной конечноразностной модели уравнения «реакция-диффузия» // Дифференц. уравнения. 1997. Т. 33, №6. С. 805-811.

2. Горчакова Е. В. Динамика слабого взаимодействия в системе близких видов // Моделирование и анализ информационных систем. 2011. Т. 18, № 1. С. 68-74.

3. Глызин С.Д., Киселева Е.О. Динамика взаимодействия пары осцилляторов нейронного типа // Моделирование и анализ информационных систем. 2008. Т. 15, № 2. C. $75-88$.

4. Глызин С.Д., Киселева Е.О. Учет запаздывания в цепочке связи между осцилляторами // Моделирование и анализ информационных систем. 2010. Т. 17, № 2. С. 140 150.

Кащенко С. А. Об одном уравнении для химеры. В работе [1] предложена следующая модель химеры:

$$
\begin{gathered}
\frac{\partial z}{\partial t}=\frac{1}{2}\left(Z-z^{2} \bar{Z}\right)+D \frac{\partial^{2} z}{\partial x^{2}}, \\
B=\int_{-L / 2}^{L / 2} d x^{\prime} G\left(\left|x-x^{\prime}\right|\right) z\left(x^{\prime}, t\right),
\end{gathered}
$$




$$
Z=B \exp \left[i \beta_{0}+i \beta_{1}|B|^{2}\right]
$$

с $L$-периодическими краевыми условиями

$$
z(x+L, t) \equiv z(x, t) .
$$

Интересом к этой модели автор обязан А. Пиковскому.

По-видимому, представляет интерес несколько иная модель, которая имеет два существенных отличия от (1)-(4).

Первое из них связано с тем, что интегрирование в (2) происходит по бесконечному промежутку

$$
B=\int_{-\infty}^{\infty} d x^{\prime} G\left(\left|x-x^{\prime}\right|\right) z\left(x^{\prime}, t\right)
$$

Важно отметить, что в этом случае, в отличие от (2), краевая задача $(1),(3),(4),(5)$ явно не зависит от пространственной переменной.

Предполагается, что значения функции $G(s)$ сосредоточены в окрестности $s=0$. В связи с этим удобно считать, что

$$
G(s)=(\sigma \sqrt{\pi})^{-1} \exp \left(-\sigma^{-2} s^{2}\right), \quad\left(\int_{-\infty}^{\infty} G(s) d s=1\right) .
$$

Отметим еще, что после стандартной замены $x \rightarrow \frac{L}{2 \pi} x$ можно перейти от (4) к $2 \pi$-периодической краевой задаче. При этом коэффициент $D$ в (1) меняется на $\varepsilon^{2} D$, а коэффициент $\sigma$ в $(6)$ на $\varepsilon \sigma$.

Рассмотрим вопрос о существовании бегущих волн - решений вида

$$
z=\rho_{k} \exp \left(i \omega_{k} t+i k x\right), \quad k=0, \pm 1, \pm 2, \ldots
$$

Подставляя (7) в (1), (3), (5), приходим к соотношениям

$$
\begin{gathered}
\exp \left(-\frac{\varepsilon^{2} \sigma^{2} k^{2}}{4}\right)\left(1-\rho_{k}^{2}\right) \cos \left(\beta_{0}+\beta_{1} \rho_{k}^{2} \exp \left(-\frac{\varepsilon^{2} \sigma^{2} k^{2}}{2}\right)\right)=2 \varepsilon^{2} k^{2} D_{1}, \\
\exp \left(-\frac{\varepsilon^{2} \sigma^{2} k^{2}}{4}\right)\left(1+\rho_{k}^{2}\right) \sin \left(\beta_{0}+\beta_{1} \rho_{k}^{2} \exp \left(-\frac{\varepsilon^{2} \sigma^{2} k^{2}}{2}\right)\right)=2 \varepsilon^{2} k^{2} D_{2}+2 \omega_{k} .
\end{gathered}
$$

При $D_{1}=0$ имеем бесконечно много корней $\rho_{k 0}=1(k=0, \pm 1, \pm 2, \ldots)$ со своими $\omega_{k 0}$ и $\rho_{k m}=\left(\exp \left(\frac{\sigma^{2} k^{2}}{2}\right)\left[\frac{\pi}{2} m-\beta_{0}\right] \beta_{1}^{-1}\right)^{1 / 2},\left(\right.$ при $\left.\left[\frac{\pi}{2} m-\beta_{0}\right] \beta_{1}^{-1}>0\right) m=2 n-1,(n=$ $0, \pm 1, \pm 2, \ldots)$, т.е. имеется последовательность $\rho_{k m} \rightarrow \infty$ при $|k| \rightarrow \infty$ и при $|m| \rightarrow \infty$.

Можно показать, что и при любом $D_{1}>0$ тоже есть сколь угодно большие корни, т.е. в моделях (1)-(4) и (1), (3), (4), (5) нет диссипативности. Можно исследовать на устойчивость каждую такую волну. При малых $\varepsilon$ имеем либо неустойчивость, либо критический случай бесконечной размерности в задаче об устойчивости.

Второе отличие от (1)-(4) обусловлено необходимостью соблюсти свойство диссипативности задачи. В этой связи представляется логичным в формулу (2) дополнительно ввести вещественную часть в коэффициент при $|B|^{2}$, т.е.

$$
Z=B \exp \left[i \beta_{0}+\left(i \beta_{1}-\mu\right)|B|^{2}\right]
$$


где $\mu>0$. Отметим, что при нахождении амплитуды бегущей волны (7) получаем уравнение, которое отличается от (9) только наличием дополнительного множителя $\exp \mu \rho^{2}$ в правой части. В этом случае уже нельзя сделать вывод об отсутствии диссипативности при $D_{1}=0$ : все значения $\rho_{k}$ находятся в некотором промежутке $\rho_{k} \leq c$ (где $c$ не зависит от $k)$. Интересно отметить, что при достаточно малых $\mu(0<\mu \ll 1)$ имеем асимптотически точную оценку

$$
c=\mu^{-1 / 2}(1+O(\mu)),
$$

а количество $N$ бегущих волн, для которых $\rho_{k}>1$, удовлетворяет асимптотически точной оценке

$$
N=2(\varepsilon \sigma)^{-1}|\ln \mu|^{1 / 2}\left(1+O\left((\ln \mu)^{-1}\right)\right) .
$$

Тем самым, с чисто математической точки зрения, имеет смысл рассматривать краевую задачу

$$
\begin{gathered}
\frac{\partial z}{\partial t}=\frac{1}{2}\left(Z-z^{2} \bar{Z}\right)+D \frac{\partial^{2} z}{\partial x^{2}}, \quad z(x+L, t) \equiv z(x, t), \\
B=(\sigma \sqrt{\pi})^{-1} \int_{-\infty}^{\infty} \exp \left(-\sigma^{-2} s^{2}\right) z(x+s, t) d s \\
Z=B \exp \left[i \beta_{0}+\left(i \beta_{1}-\mu\right)|B|^{2}\right] .
\end{gathered}
$$

1. Bordyugov G., Pikovsky A., Rosemblum M. Self-emerging and turbulent chimeras in oscillator chains // Phys. Rev. 2010. E 82. 035205 (R) (4 pages). 